\title{
SATISFACCIÓN ESTUDIANTIL CON EL USO DE OBJETOS DE APRENDIZAJE - APP EN BIOQUÍMICA EN LA CARRERA DE MEDICINA DE LA UNIVERSIDAD NACIONAL DE CHIMBORAZO (UNACH) STUDENT SATISFACTION WITH THE USE OF LEARNING OBJECTS - APP IN BIOCHEMISTRY IN THE MEDICAL CAREER OF THE NATIONAL UNIVERSITY OF CHIMBORAZO (UNACH)
}

\author{
María Angélica Barba Maggi'
}

Lucila Jazmín De la Calle Andrade ${ }^{2}$

Yisela Carolina Ramos Campi ${ }^{3}$

\section{Erika Valeria Calderón Barba ${ }^{4}$}

Gustavo Eduardo Fernández Villacré5

Diana Cristina Aguilar Marcillo ${ }^{6}$

Ecuador

1 Universidad Nacional de Chimborazo (UNACH). Riobamba, Ecuador. Docente investigadora de las carreras de Medicina y Laboratorio Clínico e Histopatológico. Doctora en Química, Magíster en Gestión Académica Universitaria. Miembro del Grupo de Trabajo en Telemedicina/Telesalud- CEDIA, Miembro Grupo TELEMED - UNACH (mbarba@unach.edu.ec). https:// orcid.org/0000-0002-3056-2974

2 Universidad Nacional de Chimborazo (UNACH). Riobamba, Ecuador. Docente investigadora de la carrera de Medicina. Doctora en Medicina y Cirugía. Magíster en Lingüística Aplicada al Aprendizaje del Inglés. Miembro del Grupo de Trabajo en Telemedicina/Telesalud- CEDIA. Miembro Grupo TELEMED - UNACH (Idelacalle@unach.edu.ec). https://orcid.org/0000-0003$\underline{2368-2027}$

3 Universidad Nacional de Chimborazo (UNACH). Riobamba, Ecuador. Docente investigadora de la carrera Laboratorio Clínico. Licenciada en Ciencias de la Salud en Laboratorio Clínico e Histopatológico, Magíster en Docencia Universitaria e Investigación Educativa. Miembro Grupo TELEMED - UNACH (yramos@unach.edu.ec). https://orcid.org/0000-0002-2403-4139

$4 \quad$ Posgrados Universidad Regional Autónoma de los Andes (UNIANDES). Ambato, Ecuador. Licenciado en Sistemas Computacionales, Doctor en Ciencias de la Educación. Miembro del Grupo de Trabajo en Telemedicina/Telesalud- CEDIA. Miembro Grupo TELEMED - UNACH (cyssaedu@hotmail.com). https://orcid.org/0000-0003-1028-1224

5 Hospital José Carrasco Arteaga. Cuenca, Ecuador. Médico General, Posgradista Imagenología Universidad de Cuenca. Miembro Grupo TELEMED - UNACH (valeria.calderonbarba@hotmail.com). https://orcid.org/0000-0002-4828-524X

6 Corporación Ecuatoriana para el Desarrollo de la Investigación y la Academia CEDIA. Cuenca, Ecuador. Ingeniera de Empresas. Coordinadora del Grupo de Trabajo Telemedicina/Telesalud- CEDIA (cristina.aguilar@cedia.org.ec). https://orcid. org/0000-0001-7400-4715 


\section{RESUMEN}

El presente trabajo, se sustentó en la investigación del grupo TELEMED (Telemedicina y Educación Médica) de la Universidad Nacional de Chimborazo (UNACH), convenios vigentes entre la universidad y Corporación Ecuatoriana para el Desarrollo de la Investigación y la Academia (CEDIA) años 2019 - 2020 e investigaciones vigentes en el grupo de Telemedicina - Telesalud reconocidos en CEDIA y registrados como proyectos externos en la UNACH. Se diseñaron Objetos de Aprendizaje en base a la metodología DICREVOA 2.0, con aplicaciones para Android (OA-app), los cuales actualmente reposan en la tienda Google Play. El Objetivo fue analizar el nivel de satisfacción con el uso de los Objetos de aprendizaje - app con tecnología Android, para el aprendizaje significativo de Bioquímica, en la temática de Hemoglobina y Mioglobina, en los estudiantes de los primeros semestres de la carrera de Medicina de la UNACH. Se trabajó con dos grupos experimentales en dos cohortes distintas, a quienes se les aplicó un cuestionario para conocer el nivel de conocimiento de OA-app y otro para determinar la satisfacción después del uso de OA-app, resultados analizados con la herramienta estadística Excel, evidenciándose que la satisfacción estudiantil después del uso de los Objetos de aprendizaje - app con tecnología Android supera el $90 \%$ de acuerdo y muy de acuerdo, en la escala valorativa más alta.

\section{PALABRAS CLAVE}

bioquímica, medicina, objeto de aprendizaje.

\section{ABSTRACT}

The present work was based on the research of the TELEMED group (Telemedicine and Medical Education) of the National University of Chimborazo (UNACH), current agreements between the university and the Ecuadorian Corporation for the Development of Research and the Academy (CEDIA), years 2019 - 2020, and current investigations in the Telemedicine - Telehealth group recognized in CEDIA and registered as external projects in $\mathrm{UNACH}$. Learning Objects were designed based on the DICREVOA 2.0 methodology, with Android applications (OA-app), which currently rest in the Google Play store. The objective was to analyze the level of satisfaction with the use of the Learning Objects - app with Android technology, for a meaningful learning of Biochemistry, on the topics of Hemoglobin and Myoglobin, in the students of the first semesters of the Medical Career of UNACH. We worked with two experimental groups in two different cohorts, to whom a questionnaire was applied to know the level of knowledge of OA-app and another to determine satisfaction after the use of OA-app, the results were analyzed with the statistical tool Excel, showing that student satisfaction after the use of the Learning Objects - app with Android technology exceeds $90 \%$ of agreement and strongly agree, on the highest rating scale.

\section{KEY WORDS}

biochemistry, medicine, learning object.

\section{INTRODUCCIÓN}

La telesalud se puede concebir como una perspectiva metodológica de amplia cobertura para desarrollar la actividad médica y educativa, al mismo tiempo que se define como un mecanismo para la administración y provisión de salud. Es posible considerar de manera holística, varias aplicaciones de las tecnologías de la información y comunicación, en áreas interconectadas, como son la educación médica y la telemedicina.

Dentro de ese espectro, surgen diferentes herramientas tecnológicas, software especializado y otros medios para ejercer la actividad de enseñanza-aprendizaje en el campo médico. El uso de las tecnologías de 
la información y comunicación se encuentra ampliamente implementado en la educación superior y en las carreras de salud, en todas las modalidades de ejecución, ya sea presencial, semipresencial y on-line. Constituyen un medio importante para el ejercicio de la docencia, que permite la interactividad y aprendizaje estudiantil, en un espacio de acción ilimitado. Las plataformas virtuales $y$ el e-learning simulan el quehacer educativo, dando solución así, a los problemas de distancia y horario para los estudiantes, contribuyendo a que el conocimiento llegue a más actores y a sitios remotos (Fernández Villacrés, Arcos Naranjo, Barba Maggi, De la Calle Andrade, \& Gualpa Jaramillo, 2016).

La influencia de la tecnología en la educación, se ha incrementado de manera exponencial, demandando mayor capacitación y compromiso de los educadores para poder ajustarla y aplicarla en sus clases y demás actividades de docencia. materiales académicos para socializarlos con sus alumnos y con otros colegas (Fernández Villacrés, Arcos Naranjo, Barba Maggi, De la Calle Andrade, \& Gualpa Jaramillo, 2016).

La introducción de las TIC en la formación médica propicia la aparición de nuevos espacios en el proceso de aprendizaje, al crear nuevos recursos e interacciones, que coadyuven al desarrollo de conocimientos y competencias (Rodríguez Beltrán, Pardo Gómez, Izquierdo La O, \& Berenguer Gouarnaluses, 2018).

Un obstáculo para el empleo continuo, deriva del costo elevado del acceso a internet y banda ancha y la infraestructura limitada de las telecomunicaciones, que se presenta en algunas regiones, siendo la tecnología móvil una vía para mejorar los índices de trasmisión de la información, sustentándose en recursos de aprendizaje en línea, tributando a los objetivos de la región de conseguir justicia y calidad en la academia (Barba Maggi, De la Calle Andrade,

Fernández Villacrés, Gualpa Jaramillo, \& Calderón Barba, 2019).

La Organización de las Naciones Unidas para la Educación, la Ciencia y la Cultura (UNESCO), en concordancia con su mandato en educación superior y de acuerdo a la meta 4.3 del Objetivo de Desarrollo Sostenible 4: "Para 2030, asegurar el acceso en condiciones de igualdad para todos los hombres y las mujeres a una formación técnica, profesional y superior de calidad, incluida la enseñanza universitaria" (Organización de las Naciones Unidas para la Educación, la Ciencia y la Cultura. UNESCO, 2021).

En virtud de lo expuesto, se evidencia la pertinencia de desarrollar herramientas tecnológicas adecuadas a los requerimientos educativos, como son los "objetos de aprendizaje digitales" y lograr su implementación a través de aplicaciones móviles para Android.

Un Objeto de Aprendizaje (OA) puede definirse como un módulo didáctico digital construido para llegar a una meta de aprendizaje simple, que tiene como característica ser reutilizable en distintos Entornos Virtuales de Enseñanza y Aprendizaje, y en variados contextos educativos. El origen del término "Objeto de Aprendizaje", se adjudica a Wayne Hodgins en 1992, quien planteó elaborar el contenido de aprendizaje digital a partir de unidades autónomas y reutilizables, susceptibles de agruparse en elementos cada vez más complejos, comparable con las construcciones de los bloques LEGO (Maldonado, 2015).

Los OA, sus aplicaciones y utilidades, han sido estudiados en diferentes trabajos de investigación y en combinación con diferentes tecnologías. En las Facultades de Ciencias de la Educación de las universidades de Sevilla y Huelva (España), se analizó la producción de objetos de aprendizaje por parte de los estudiantes, en realidad aumentada, concluyendo que su uso 
en la enseñanza universitaria ha despertado un alto grado de aceptación y motivación (Barroso, Cabero, \& Gutiérrez, 2018).

La comunicación entre docentes y alumnos se puede ver fortalecida mediante el desarrollo de objetos de aprendizaje en entornos y campus virtuales, como lo demuestran Juan Cabanillas García (2019) y su equipo de investigación en el trabajo realizado en la Universidad de Extremadura, cuyo objetivo fue analizar los objetos de aprendizaje y la percepción del profesorado sobre el campus virtual, señalando además, que los principales aspectos negativos detectados fueron su funcionamiento y su interfaz, sin que existan variaciones significativas por cuestión de género, edad y rama de investigación (Cabanillas, Luengo, \& Carvalho, 2019).

Así mismo, corresponde resaltar la necesidad inminente de capacitar al profesorado en el área del diseño de objetos de aprendizaje y otras tecnologías. Las instituciones de educación, encuentran necesario ofertar cursos de formación en prácticas de codiseño y diseño de recursos didácticos digitales, para enfrentar adecuadamente los avances en la transferencia del conocimiento (Diez \& Morales, 2020)

En la Universidad Nacional de Chimborazo (UNACH), se apoya continuamente la formación docente en uso de recursos y metodologías de aprendizaje. Como parte de la investigación desarrollada en red con la Corporación Ecuatoriana para el Desarrollo de la Investigación y la Academia (CEDIA), el grupo de Telemedicina y Educación Médica, desarrolló una Capacitación en el diseño de objetos de aprendizaje para la actualización en recursos didácticos con sustento en la Tic, demostrándose que la aplicación de objetos de aprendizaje en un grupo experimental, favorece la obtención de mejores resultados educativos. Se concluye la importancia de la capacitación, actualización y perfeccionamiento de los profesores en el diseño de recursos didácticos OA (Barba, De la Calle, Calderón, \& Ramos, 2019)

En esta misma línea de estudio, se han obtenido significativos resultados sobre el uso y aplicación de OA:

- Durante el año 2016, en la carrera de Medicina de la Universidad Nacional de Chimborazo (UNACH), mediante la aplicación de Objetos de Aprendizaje (OA) estructurados a los estudiantes de Neurología, se obtuvo como conclusión que la utilización de recursos educativos virtuales, planificados y orientadores, como son los OA, diseñados conforme al contenido del sílabo, en el abordaje de la cátedra de Neurología aplicada al Trauma, permiten tener un conocimiento más efectivo que solamente los recursos tradicionales.

- Se ha evidenciado que la aplicación en el plan de estudio de los OA, conduce a una mayor satisfacción con el proceso $(90 \%$ de los estudiantes manifestaron su satisfacción y su alto grado de participación).

- La aplicación de OA como estrategia académica, contribuye significativamente al aprendizaje de la Bioquímica I, en una escala cualitativa de Muy Bueno (A-).

- Los Objetos de Aprendizaje 3D son herramientas empleados en el presente, para alcanzar una formación individualizada y comunicativa, acorde a las necesidades de aprendizaje, en la cátedra de Anatomía IV (Neuroanatomía), determinándose que un $74.32 \%$ de estudiantes alcancen dominar los contenidos desde las aulas virtuales (Barba, De la Calle, \& Fernández, Aplicación de objetos de aprendizaje 3D en Neuroanatomía, 2018) 
La estructura general de un objeto de aprendizaje

(OA) se representa en el siguiente esquema

(Figura 1):

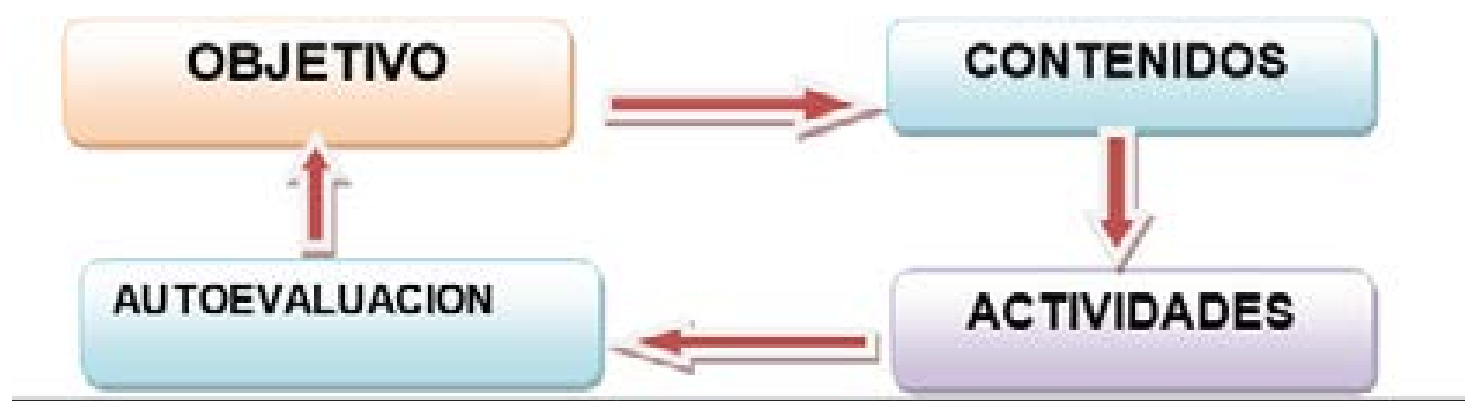

Figura 1. Estructura general de un objeto de aprendizaje.

Fuente: Objetos de aprendizaje para la enseñanza de Telemedicina en las Universidades: UNIANDES, UTA y UNACH (Fernández Villacrés, Arcos Naranjo, Barba Maggi, De la Calle Andrade, \& Gualpa Jaramillo, 2016)

Para la elaboración de los mismos se tuvieron en cuenta los siguientes criterios:

- Metodología de desarrollo: DICREVOA 2.0

- Software a utilizar: Exelearning (Herramienta de autor de código abierto "open source".

- La plantilla para el diseño de los objetos de aprendizaje, estableció los siguientes componentes: Descripción textual del contenido, Objetivo de aprendizaje, Contenidos, Actividades, Autoevaluación, Diseño Multimedia, Diseño de la interfaz, Estructura de las pantallas, Navegación (Fernández Villacrés, Arcos Naranjo, Barba Maggi, De la Calle Andrade, \& Gualpa Jaramillo, 2016)

Por otro lado, el uso de las aplicaciones móviles (APP) en salud y educación, ha ganado terreno para lograr la captación del mayor número de individuos en diversos procesos. Se debe considerar los procedimientos de regulación a diferente nivel de las aplicaciones móviles en salud (mHealth), tanto para utillización profesional como por parte de pacientes, y otras aplicaciones destinadas hacia la prevención (Alonso \& Mirón, 2017). De igual forma, el uso de las APPS en la educación (m-Learning) ha sido evaluado en sus diferentes aspectos.

En el trabajo realizado por Javier Fombona, María Pascual y Esteban Vázquez (2020), "M-Learning en niveles iniciales, rasgos didácticos de las APPS educativas", entre sus conclusiones, se indica que el m-Learning, además de sus utilidades educativas y para adquisición de información, tiene una importancia cultural y social, constituyéndose en un nuevo reto, ante los estudiantes contemporáneos, la autonomía en el aprendizaje, el uso extendido de la tecnología y la mayor conectividad (Fombona , Pascual, \& Vásquez-Cano, 2020).

El uso de aplicaciones móviles promueve el desarrollo de determinadas habilidades referentes a la innovación docente y emprendimiento. Por ejemplo, el uso de la gamificación en conjunto con aplicaciones móviles, que constituye una novedosa estrategia para intervenir y promover la participación de distintos actores en las comunidades y redes 
sociales (García, Fernández, Sánchez, \& Grimaldi, 2017)

Al diseñar aplicaciones móviles, es importante tomar en cuenta aspectos diversos sobre su empleo generalizado. Tales condiciones implican los adelantos en técnicas y métodos que los desarrolladores deben desplegar para probar el uso de las APPS, con el fin de reducir el peligro de fallas, y así lograr la confianza en los consumidores finales (Velásquez, Monsalve, Zapata, Gómez, \& Ríos, 2019).

Las APPS tienen una gran influencia en los estudiantes de nuevas generaciones, por lo que su acogida y aprobación dentro del proceso educativo es significativa. El estudio ejecutado en la Universidad Tecnológica de Tulancingo, evidencia que el $95 \%$ de los alumnos encuestados expresan su aceptación para el uso de una aplicación móvil gratuita como "refuerzo educativo", siendo una opción aprovechar al máximo este entorno virtual que $\begin{array}{llll}5 & 1 & 7 & \text { los estudiantes jóvenes incluyen en su realidad }\end{array}$ (Cárdenas \& Cáceres, 2019)

Existen diversas plataformas para móviles iPhone, Symbian, Windows, BlackBerry, Palm, Java Mobile Edition, Linux Mobile (LiMo), Android- en uso hoy en día. Cabe mencionar que Android muestra una sucesión de características que lo hacen diferente (Barba Maggi, De la Calle Andrade, Fernández Villacrés, Gualpa Jaramillo, \& Calderón Barba, 2019). Es el primero que fusiona en un mismo recurso las siguientes cualidades: Plataforma realmente abierta, portabilidad asegurada, arquitectura basada en componentes inspirados en Internet, filosofía de dispositivo siempre conectado a Internet, gran cantidad de servicios incorporados, alto nivel de seguridad, optimización para baja potencia y poca memoria, alta calidad de gráficos y sonidos (Gironés, 2013).

La ejecución de proyectos de investigación, tales como "Aplicaciones móviles con tecnología
Android para la enseñanza de Bioquímica y Medicina en las Universidades UNIANDES, UNACH, UTB y UCACUE" patrocinado por CEDIA y realizado por las instituciones en mención, ha permitido establecer la problemática concerniente con el insuficiente uso de las aplicaciones móviles como componentes de soporte didáctico en el aprendizaje de Medicina. Se ha encontrado que la mayoría de los estudiantes tienen acceso a teléfonos inteligentes con sistema operativo Android. En virtud de los resultados parciales de este estudio, se desarrollaron varias aplicaciones móviles para el área de Bioquímica, ubicadas en la tienda de Google play, para la respectiva descarga (Barba Maggi, De la Calle Andrade, Fernández Villacrés, Gualpa Jaramillo, \& Calderón Barba, 2019)

Con estos antecedentes, se ha realizado la presente investigación con el objetivo de analizar el nivel de satisfacción con el uso de los Objetos de aprendizaje - app con tecnología Android, para el aprendizaje significativo de Bioquímica, en la temática de Hemoglobina y Mioglobina, en los estudiantes de los primeros semestres de la carrera de Medicina de la UNACH.

\section{METODOLOGÍA}

\section{Contexto de estudio}

En la Universidad Nacional de Chimborazo, Facultad de Ciencias de la Salud, carrera de Medicina el rediseño curricular vigente desde el año 2017, aprobado por el Consejo de Educación Superior (CES) mediante resolución RPC-SO-39-No.726-2017 (Consejo de Educación Superior, 2017), contiene en su meso currículo la asignatura de Bioquímica I, con una dedicación de 5 horas semanales distribuidas 2 para el componente docente, 3 para las prácticas de aplicación y experimentación de los aprendizajes y 1 para el componente de trabajo autónomo, en el microcurrículo de la Unidad 2 se 
aborda el estudio de los Aminoácidos, Péptidos y Proteína, modalidad presencial con soporte en el uso de aula virtuales, los temas abordados en la presente investigación fueron Hemoglobina y Mioglobina.

\section{Población y Muestra}

La población y muestra en el presente análisis correspondió a los estudiantes matriculados, en la carrera de Medicina de la UNACH de primeros semestres paralelos $B$, que fueron parte de los grupos experimentales a los que se les aplicó en su proceso académico Objetos de aprendizaje - app con tecnología Android (OA-app) indistintamente en dos periodos académicos marzo - agosto 2019 y octubre 2019 - marzo 2020 se indica en la Tabla 1.

\section{POBLACION Y MUESTRA}

Estudiantes primer semestre Bioquímica I Medicina paralelo B marzo - agosto 2019

Estudiantes primer semestre Bioquímica I Medicina paralelo B octubre 2019 - marzo 2020

TOTAL ESTUDIANTES

Tabla 1. Análisis de la población y muestra.

Ecuador, 2019.

Fuente: Nómina Estudiantes Sistema Control Académico (SICOA) UNACH. Elaboración propia.

518 . Los 60 estudiantes que corresponden al $100 \%$, durante los periodos de la investigación alcanzaron un resultado de aprendizaje que se resume en la Tabla 2 y respondieron al instrumento diseñado y validado para la investigación.

\section{RESULTADO DE LA EVALUACIÓN DEL APRENDIZAJE DE BIOQUÍMICA: HEMOGLOBINA Y} MIOGLOBINA

\begin{tabular}{|c|c|c|c|c|c|c|c|}
\hline \multirow{2}{*}{ Escala } & \multicolumn{3}{c|}{ (CORTE I) } & \multicolumn{3}{c|}{ (CORTE II) } \\
\hline Cualitativa & Cuantitativa & f.i. & marzo - agosto 2019 & \multicolumn{2}{c|}{ octubre 2019 - marzo 2020 } \\
\hline Excelente (A+) & 10,0 & 0 & 0,00 & 0 & 0 & 0,00 & F.i \\
\hline Excelente (A) & $9,5-9,9$ & 25 & 67,57 & 25 & 2 & 8,70 & 2 \\
\hline Muy Bueno (A-) & $9,0-9,4$ & 11 & 29,73 & 36 & 11 & 47,83 & 13 \\
\hline Muy Bueno (A) & $8,5-8,9$ & 0 & 0,00 & 36 & 9 & 39,13 & 22 \\
\hline Bueno (B) & $8,0-8,4$ & 1 & 2,70 & 37 & 1 & 4,35 & 23 \\
\hline Bueno (B-) & $7,5-7,9$ & 0 & 0,00 & & 0 & 0,00 & \\
\hline Aprobado (C) & $7-7,4$ & 0 & 0,00 & & 0 & 0,00 & \\
\hline No Aprobado (D) & $<6,9$ & 0 & 0,00 & & 0 & 0,00 & \\
\hline TOTAL & & $\mathbf{3 7}$ & $\mathbf{1 0 0}$ & & $\mathbf{2 3}$ & $\mathbf{1 0 0}$ & \\
\hline
\end{tabular}

Tabla 2. Evaluación del aprendizaje.

Ecuador, 2019-2020. Fuente: Actas de Calificaciones Asignatura Bioquímica I Medicina - UNACH periodos marzo - agosto 2019 y octubre 2019 - marzo 2020. Elaboración propia. 


\section{Variables Estratégicas instruccionales}

Las estrategias contribuyen al cumplimiento de logros, objetivos o competencias. En el campo académico, el proceso de aprendizaje es el objeto de las estrategias instruccionales, ejecutadas por los estudiantes en ausencia o presencia de los docentes (Díaz, Miguel, Landaeta, Ortiz, \& Fernández, 2014)
Estrategias instruccionales implementadas en la UNACH y como parte de la libertad de cátedra asumida de forma responsable y que se apoyan en el uso de la Tecnología de la información y comunicación (Tic), que contribuyen al logro de resultados de aprendizaje descritos en la planificación microcurricular, se resumen de forma general en la metodología de la asignatura de Bioquímica I, con y sin Tic. Tabla 3.

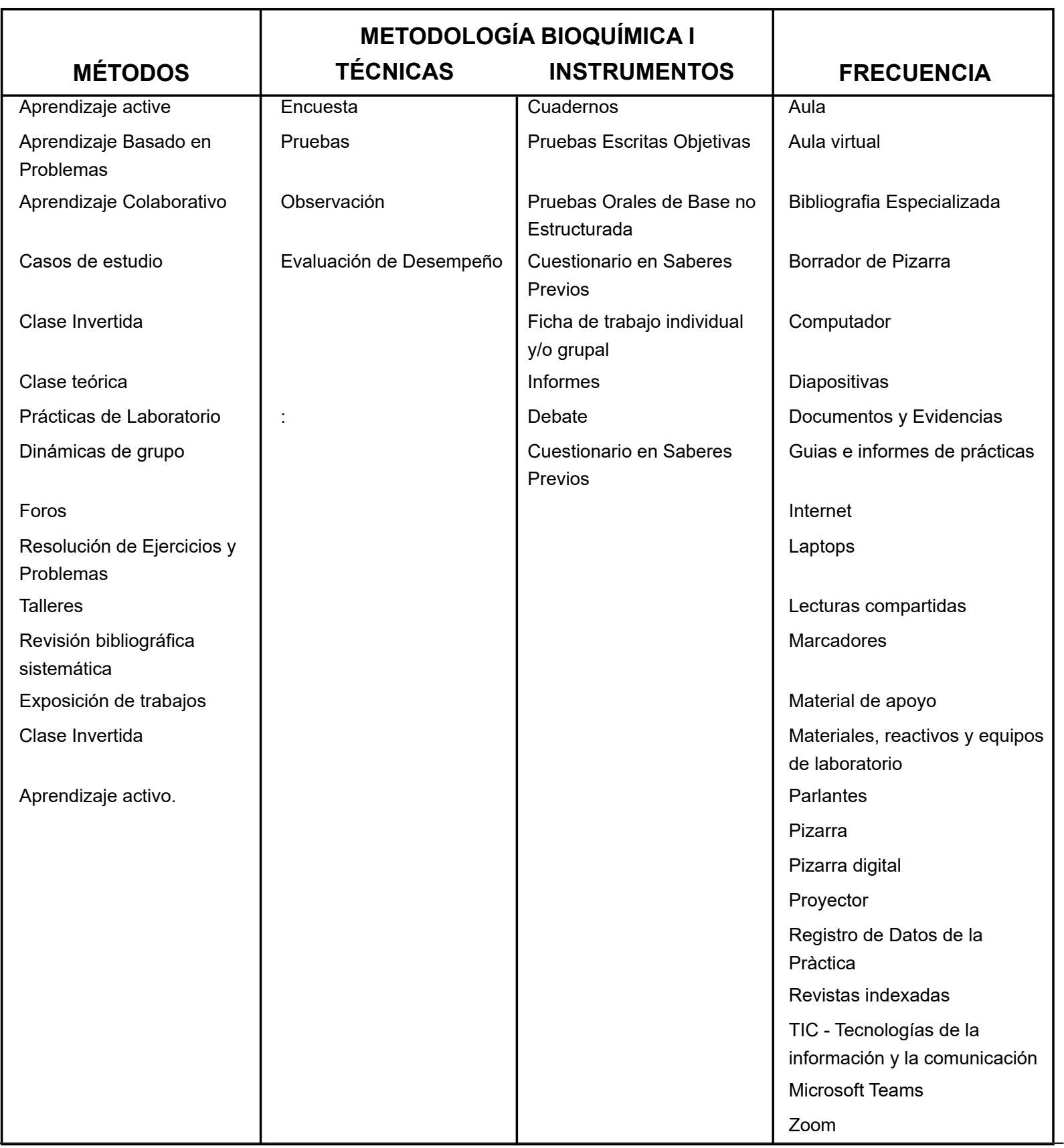

Tabla 3. Metodología utilizada para el aprendizaje de la asignatura de Bioquímica II.

Fuente: Sílabo Asignatura Bioquímica I (SICOA) UNACH. Elaboración propia. 
Fueron cuatro OA-app utilizados como estrategias o recursos digitales, para el proceso de aprendizaje teórico práctico, de los temas Hemoglobina y Mioglobina que se aplicaron, las figuras 2, 3, 4 y 5 de estos recursos digitales constan en la tienda Google Play diseñados
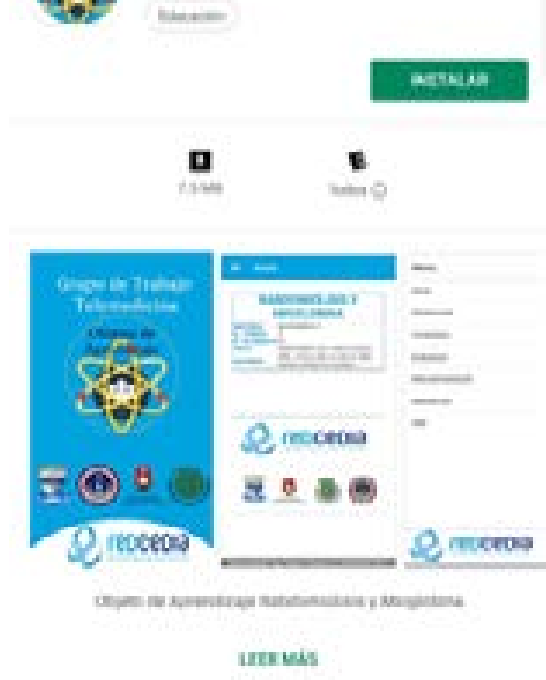

Cellowe nowationide

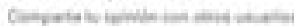

OA Trauma y Ilemeglobina haven men a en base a la metodología de desarrollo: Metodología de desarrollo: DICREVOA 2.0, software utilizado: Exelearning (Herramienta de autor de código abierto "open source") y con aplicación app para Sistema Operativo Android.
OA CunnEficesión Migglobing

fermiliomis

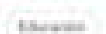

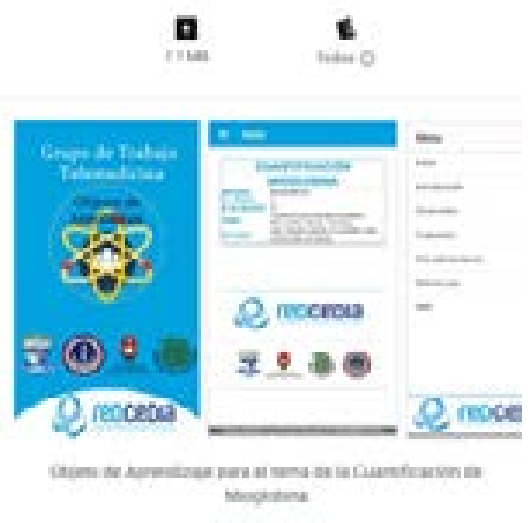

ursvein

Caifear esta wescavide

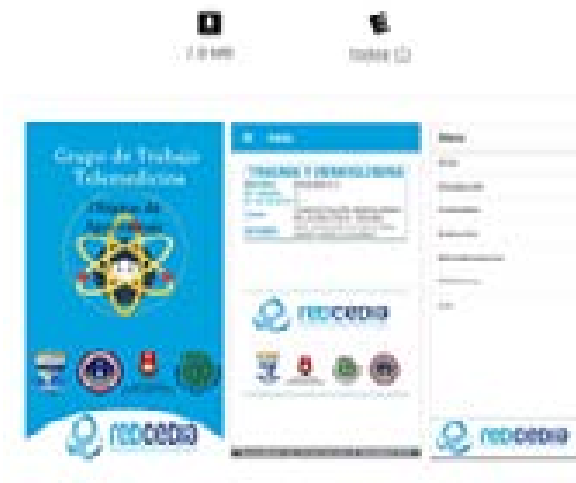

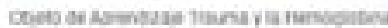

ctenass
OA Cuantificación Homoglobina

Foublutum
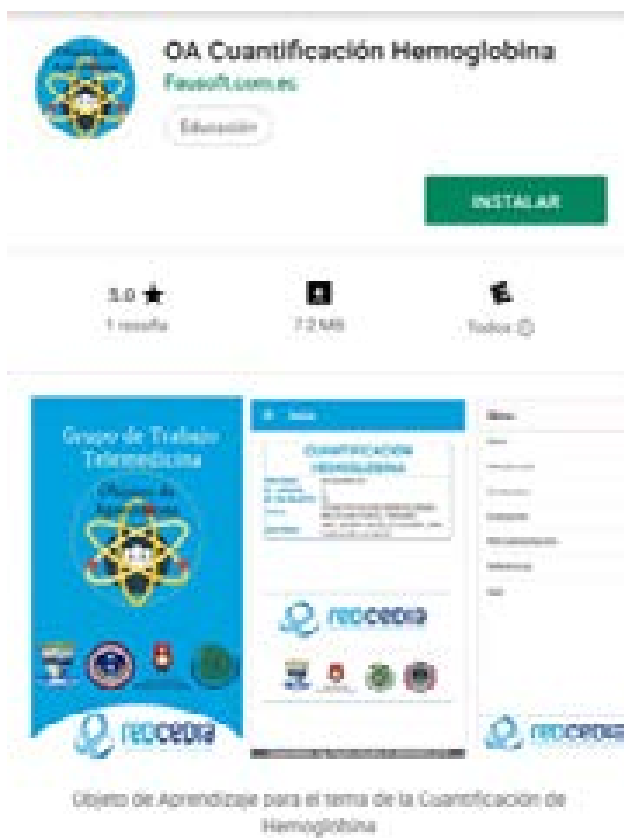

Cakfour este aplitsoiton

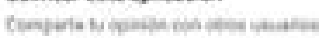

Figura 2. Objetos de Aprendizaje - app. Ecuador, 2019

Fuente: Tienda de Google Play. Elaboración propia. 
Después de la utilización de los OA-app se evalúa el resultado de aprendizaje y se mide el nivel de satisfacción estudiantil, definida como una sensación o estado de ánimo que manifiestan los estudiantes, posterior a cumplir sus expectativas y requerimientos (Díaz, Miguel, Landaeta, Ortiz, \& Fernández, 2014). Por ejemplo, se puede usar el instrumento presentado en el artículo "Un Estudio de la Satisfacción Obtenida con el Uso de Objetos de Aprendizaje" (Velázquez, y otros, 2014) y en "Aplicación de objetos de aprendizaje 3D en Neuroanatomía" (Barba, De la Calle, \& Fernández, Aplicación de objetos de aprendizaje 3D en Neuroanatomía, 2018)

\section{INSTRUMENTOS}

Los instrumentos fueron diseñados $y$ validados por expertos en el área y aplicados en investigaciones anteriores del grupo de profesionales que presentan este análisis. Los citados instrumentos se aplicaron de forma anónima y voluntaria a los estudiantes participantes. Un primer cuestionario permitió recabar información del conocimiento de OAapp e información general de los estudiantes se indican las preguntas y opciones de respuesta en la Tabla 4. El segundo cuestionario se aplicó después de la utilización de los OA-app y de la emisión de resultados de aprendizaje, las peguntas del instrumento para determinar la satisfacción con el uso de OA-app se resumen en la Tabla 5.

\begin{tabular}{|c|c|c|}
\hline \multicolumn{3}{|c|}{ Cuestionario para determinar conocimiento de OA-app } \\
\hline & Pregunta & Opciones de Respuesta \\
\hline 1 & Dispositivo móvil utiliza & Smart $(\ldots) \quad$ Sencillo $(\ldots)$ \\
\hline 2 & Sistema operativo del dispositivo móvil & $\begin{array}{l}\text { Android }(\ldots) \quad \text { los }(\ldots) \text { Windows }(\ldots) \\
\text { Desconozco }(\ldots)\end{array}$ \\
\hline 3 & Conexión permanente a Internet & Si $(\ldots) \quad$ No $(\ldots) \quad$ Me es indiferente $(\ldots)$ \\
\hline 4 & $\begin{array}{l}\text { Dispositivo móvil lo utiliza como elemento de } \\
\text { apoyo didáctico }\end{array}$ & Si $(\ldots) \quad$ No $(\ldots)$ Me es indiferente $(\ldots)$ \\
\hline 5 & Conoce para qué sirve el lector QR & Si $(\ldots) \quad$ No $(\ldots)$ Me es indiferente $(\ldots)$ \\
\hline 6 & El lector QR lo utiliza en & $\begin{array}{l}\text { Aplicaciones móviles }(\ldots) \quad \text { whatsapp }(\ldots) \\
\text { mensajes }(\ldots) \quad \text { No lo utiliza }(\ldots)\end{array}$ \\
\hline 7 & $\begin{array}{l}\text { Cree que la tendencia de la tecnología es } \\
\text { hacia el uso de }\end{array}$ & $\begin{array}{l}\text { Uso de dispositivos móviles }(\ldots) \\
\text { Computadoras portátiles }(\ldots) \text { Computadoras } \\
\text { de escritorio }(\ldots) \text { Tablets }(\ldots)\end{array}$ \\
\hline 8 & $\begin{array}{l}\text { Ha utilizado objetos de aprendizaje apps en su } \\
\text { actividad académica }\end{array}$ & si $(\ldots) \quad$ no $(\ldots)$ me es indiferente $(\ldots)$ \\
\hline 9 & $\begin{array}{l}\text { En el currículo de medicina uso de objetos } \\
\text { digitales de aprendizaje como apoyo } \\
\text { pedagógico }\end{array}$ & No $(\ldots)$ Me es indiferente $(\ldots)$ \\
\hline 10 & $\begin{array}{l}\text { Docentes y estudiantes de medicina utilizan } \\
\text { recursos digitales para el proceso de } \\
\text { enseñanza y aprendizaje }\end{array}$ & Si $(\ldots) \quad$ No $(\ldots) \quad$ M es indiferente $(\ldots)$ \\
\hline
\end{tabular}




\begin{tabular}{|c|c|c|}
\hline 11 & $\begin{array}{l}\text { Cree que las aplicaciones móviles pueden } \\
\text { convertirse en el principal elemento de apoyo } \\
\text { educativo por su movilidad }\end{array}$ & Si $(\ldots) \quad$ No $(\ldots)$ Me es indiferente $(\ldots)$ \\
\hline 12 & $\begin{array}{l}\text { Aplicar estrategias con el fin de incrementar } \\
\text { el uso de recursos digitales e implementar las } \\
\text { aplicaciones móviles como apoyo educativo }\end{array}$ & $\begin{array}{l}\text { Nunca }(\ldots) \quad \text { Rara vez }(\ldots) \\
\text { Frecuentemente }(\ldots) \quad \text { Siempre }(\ldots)\end{array}$ \\
\hline 13 & $\begin{array}{l}\text { Debe propender la realización de eventos de } \\
\text { capacitación sobre el uso de recursos digitales } \\
\text { y aplicaciones móviles como apoyo educativo, } \\
\text { dirigidos a docentes }\end{array}$ & Si $(\ldots) \quad$ No $(\ldots)$ Me es indiferente $(\ldots)$ \\
\hline
\end{tabular}

Tabla 4. Cuestionario aplicado para determinar conocimiento de OA-app. Ecuador, 2019-2020

Fuente: Encuesta Aplicada Estudiantes Bioquímica I - UNACH periodos marzo - agosto 2019 y octubre 2019 - marzo 2020. Elaboración propia.

\begin{tabular}{|c|l|}
\hline \multicolumn{2}{|c|}{ Cuestionario para determinar la Satisfacción en el uso de OA-app } \\
\hline 1 & El tema de presentación es fácil de entender \\
\hline 2 & Los objetos de aprendizaje app permiten mejor compresión del tema \\
\hline 3 & La presentación e imágenes son creativas y atractivas \\
\hline 4 & El objeto de aprendizaje app permite el trabajo colaborativo y en equipo \\
\hline 5 & El objeto de aprendizaje app de Bioquímica tiene bibliografía actualizada \\
\hline 6 & En el objeto de aprendizaje app se utiliza un lenguaje adecuado y consecuente con la medicina \\
\hline 7 & Se evidencia investigación profunda del tema \\
\hline 8 & Optimiza mi tiempo en la búsqueda de información sobre el tema tratado \\
\hline 9 & Me ayudo en motivarme a la investigación el tema \\
\hline 10 & Recomendaría el trabajo con los objetos de aprendizaje app \\
\hline 11 & Los profesores conocen del tema para el manejo de estos recursos \\
\hline 12 & Se integra completamente el trabajo teórico y práctico al utilizar objetos de aprendizaje app \\
\hline 13 & Quiero continuar utilizando estos recursos en mis clases \\
\hline
\end{tabular}

Tabla 5. Cuestionario aplicado para determinar la Satisfacción en el uso de OA-app

Ecuador, 2019-2020

Fuente: Encuesta Aplicada Estudiantes Bioquímica I - Medicina - UNACH periodos marzo - agosto 2019 y octubre 2019 - marzo 2020. Elaboración propia.

La escala ordinal utilizada para analizar la Satisfacción en el uso de OA-app fue Muy de acuerdo (MA), Acuerdo (A), Neutral (N), Desacuerdo (DSA), Muy en desacuerdo (MSDA).

\section{RESULTADOS Y DISCUSIÓN}

Los datos obtenidos se tabulan y se analizan con la herramienta Excel.
Entre varios de los requerimientos para la utilización de Tic y de los Objetos de aprendizaje - app, están el disponer de equipos para conexión como tablets, celulares con un sistema operativo adecuado, portátiles, contar con acceso a internet, manejar recursos digitales, objetos de apendizaje, conocer de lectores $\mathrm{QR}$, etc. 
Al analizar los resultados del conocimiento de $O A$-app que contribuyen a responder algunas interrogantes se evidencia que, de los 60 estudiantes que corresponden al 100\%, 95\% utilizan dispositivos móviles Smart y el 5\% Sencillo; el $85 \%$ tienen un Sistema operativo Android, $9.67 \%$ los, el 1,67\% Windows en su dispositivo móvil., el $85 \%$ dispone de forma permanente conexión a internet y el $7.5 \%$ no disponen., el 93,33\% de estudiantes emplean su dispositivo móvil como apoyo didáctico y el $6,67 \%$ no lo utilizan., el 81,67\% conocen para sirven los lectores $\boldsymbol{Q} \boldsymbol{R}$, el $13,33 \%$ no conocen y al $5 \%$ le es indiferente., el 38,33\% utilizan el lector $\mathbf{Q R}$ en aplicaciones móviles, el $21,88 \%$ para WhatsApp, $5,47 \%$ en mensajes y el $1,56 \%$ no lo utiliza., el $88,33 \%$ consideran que la tendencia de la tecnología es al uso de dispositivos móviles, el 6,67\% computadoras portátiles, 3,33\% computadoras de escritorio, $1,67 \%$ tablets., el $96,67 \%$ de estudiantes usa objetos de aprendizaje con android en la actividad académica, el 3,33\% no lo usan., el 90 $\%$ de estudiantes consideran que si deben utilizar en el currículo de medicina objetos digitales de aprendizaje como apoyo pedagógico, el $10 \%$ dicen que no., el $23,33 \%$ indican que docentes y estudiantes de medicina docentes $y$ estudiantes de medicina utilizan recursos digitales para el proceso de enseñanza y aprendizaje, el $73,33 \%$ que no utilizan y el $3,33 \%$ indican que no., el 93,33\% indican que las aplicaciones móviles pueden convertirse en el principal elemento de apoyo educativo por su movilidad el 6,67\% consideran que no, consideran que aplicar estrategias con el fin de incrementar el uso de recursos digitales e implementar las aplicaciones móviles como apoyo educativo el 3,33 \% nunca, $23,33 \%$ rara vez, el $60 \%$ indican que frecuentemente y el $13,33 \%$ siempre., el $86,67 \%$ indican que deben realizarse eventos de capacitación sobre el uso de recursos digitales $y$ aplicaciones móviles como apoyo educativo, dirigidos a docentes, $8.33 \%$ dicen que no para el $5 \%$ les es indiferente.

La aplicación del cuestionario para determinar la Satisfacción en el uso de OA-app permitió obtener los resultados, que se presentan en la TABLA 6.

\begin{tabular}{|c|l|}
\hline & \multicolumn{1}{|c|}{ Análisis Resultados de Satisfacción en el uso de OA-app } \\
\hline $\mathbf{1}$ & $\begin{array}{l}\text { El } 95 \% \text { del grupo experimental expresa estar de acuerdo y muy de acuerdo en que el tema es } \\
\text { fácil de entender incluido en punto neutro de valoración }\end{array}$ \\
\hline $\mathbf{2}$ & $\begin{array}{l}\text { El } 95 \% \text { del grupo experimental expresa estar de acuerdo y muy de acuerdo que los objetos de } \\
\text { aprendizaje app permiten mejor compresión del tema incluido en punto neutro de valoración }\end{array}$ \\
\hline $\mathbf{3}$ & $\begin{array}{l}\text { El } 95 \% \text { del grupo experimental expresa estar de acuerdo y muy de acuerdo la presentación e } \\
\text { imágenes son creativas y atractivas incluido en punto neutro de valoración }\end{array}$ \\
\hline $\mathbf{4}$ & $\begin{array}{l}\text { El } 95 \% \text { del grupo experimental expresa estar de acuerdo y muy de acuerdo que el objeto } \\
\text { de aprendizaje app permite el trabajo colaborativo y en equipo incluido en punto neutro de } \\
\text { valoración }\end{array}$ \\
\hline $\mathbf{5}$ & $\begin{array}{l}\text { El } 91.67 \% \text { del grupo experimental expresa estar de acuerdo y muy de acuerdo en que el tema } \\
\text { es fácil de entender incluido en punto neutro de valoración }\end{array}$ \\
\hline $\mathbf{6}$ & $\begin{array}{l}\text { El } 95 \% \text { del grupo experimental expresa estar de acuerdo y muy de acuerdo en que el objeto } \\
\text { de aprendizaje app de Bioquímica tiene bibliografía actualizada incluido en punto neutro de } \\
\text { valoración }\end{array}$ \\
\hline
\end{tabular}




\begin{tabular}{|c|l|}
\hline $\mathbf{7}$ & $\begin{array}{l}\text { El } 95 \% \text { del grupo experimental expresa estar de acuerdo y muy de acuerdo en que se } \\
\text { evidencia investigación profunda del tema incluido en punto neutro de valoración }\end{array}$ \\
\hline $\mathbf{8}$ & $\begin{array}{l}\text { El } 91.67 \% \text { del grupo experimental expresa estar de acuerdo y muy de acuerdo en que optimiza } \\
\text { el tiempo en la búsqueda de información sobre el tema tratado incluido en punto neutro de } \\
\text { valoración }\end{array}$ \\
\hline $\mathbf{9}$ & $\begin{array}{l}\text { El } 98.33 \% \text { del grupo experimental expresa estar de acuerdo y muy de acuerdo en que le } \\
\text { ayuda en motivarse a la investigación el tema incluido en punto neutro de valoración }\end{array}$ \\
\hline $\mathbf{1 0}$ & $\begin{array}{l}\text { El } 100 \% \text { del grupo experimental expresa estar de acuerdo y muy de acuerdo en que } \\
\text { recomendaría el trabajo con los objetos de aprendizaje app incluido en punto neutro de } \\
\text { valoración }\end{array}$ \\
\hline $\mathbf{1 1}$ & $\begin{array}{l}\text { El } 100 \% \text { del grupo experimental expresa estar de acuerdo y muy de acuerdo en que los } \\
\text { profesores conocen del tema para el manejo de estos recursos incluido en punto neutro de } \\
\text { valoración }\end{array}$ \\
\hline $\mathbf{1 2}$ & $\begin{array}{l}\text { El } 95 \% \text { del grupo experimental expresa estar de acuerdo y muy de acuerdo en que se integra } \\
\text { completamente el trabajo teórico y práctico al utilizar objetos de aprendizaje app incluido en } \\
\text { punto neutro de valoración }\end{array}$ \\
\hline $\mathbf{1 3}$ & $\begin{array}{l}\text { El } 100 \% \text { del grupo experimental expresa estar de acuerdo y muy de acuerdo en quieren } \\
\text { continuar utilizando estos recursos en mis clases incluido en punto neutro de valoración }\end{array}$ \\
\hline
\end{tabular}

Tabla 6. Resultados de Satisfacción en el uso e OA-app

Ecuador, 2019-2020

Fuente: Resultados de Cuestionario aplicado a Estudiantes Bioquímica I Medicina - UNACH periodos marzo - agosto 2019 y octubre 2019 - marzo 2020. Elaboración propia.

El porcentaje de satisfacción después del uso de los OA-app en 11 de las preguntas supera el $95 \%$ y en 2 el $90 \%$ en las opciones de acuerdo y muy de acuerdo, en la escala más alta valorativa.

En el estudio realizado por Díaz, K., Miguel, V., Landaeta, I., Ortiz, M., \& Fernández, M. (2014) "Satisfacción estudiantil con el uso de estrategias instruccionales apoyadas en TIC para el aprendizaje de Bioquímica" concluye que "todas las estrategias instruccionales fueron consideradas como satisfactorias por el 80 $\%$ o más de los encuestados" (Díaz, Miguel, Landaeta, Ortiz, \& Fernández, 2014), hay una relación directa con el resultado obtenido en la presente investigación que determina que la satisfacción estudiantil después del uso de los OA-app en 11 de las preguntas supera el $95 \%$ y en 2 el $90 \%$ en las opciones de acuerdo y muy de acuerdo, en la escala valorativa más alta.

\section{CONCLUSIONES}

EL 95\% de estudiantes utilizan dispositivos móviles Smart; el $85 \%$ tienen un Sistema operativo Android, el $85 \%$ dispone de forma permanente conexión a internet., el 93,33\% de estudiantes emplean su dispositivo móvil como apoyo didáctico, el 81,67 \% conocen para sirven los lectores QR,.., el 38,33\% utilizan el lector QR en aplicaciones móviles, el 21,88\% para WhatsApp, $5,47 \%$ en mensajes y el $1,56 \%$ no lo utiliza., el $88,33 \%$ consideran que la tendencia de la tecnología es al uso de dispositivos móviles, el 96,67 \% de estudiantes usa objetos de aprendizaje con android en la actividad académica, el $90 \%$ de estudiantes consideran que si deben utilizar en el currículo de medicina objetos digitales de aprendizaje como apoyo pedagógico, el $73,33 \%$ indican que docentes y estudiantes de medicina docentes y estudiantes de medicina no utilizan recursos digitales para 
el proceso de enseñanza y aprendizaje, el 93,33 $\%$ indican que las aplicaciones móviles pueden convertirse en el principal elemento de apoyo educativo por su movilidad, el $86,67 \%$ indican que deben realizarse eventos de capacitación sobre el uso de recursos digitales y aplicaciones móviles como apoyo educativo, dirigidos a docentes.

La satisfacción estudiantil después del uso de los Objetos de aprendizaje - app con tecnología Android supera el $90 \%$ de acuerdo y muy de acuerdo, en la escala valorativa más alta, lo que posibilita continuar con el uso de este tipo de estrategias académicas fundamentadas en la Tecnología de la Información y comunicación.

Con la presente investigación se evidencia que el diseño y aplicación de los Objetos de aprendizaje - app dentro de una ciencia básica como en la Bioquímica, contribuye en el proceso de aprendizaje, es importante que el estudiante trabaje con un alto nivel de satisfacción para

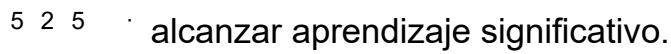

\section{REFERENCIAS BIBLIOGRÁFICAS}

Alonso, J., \& Mirón, J. A. (2017). Aplicaciones móviles en salud: potencial, normativa de seguridad y regulación. Revista Cubana de Información en Ciencias de la Salud, 28(3). Recuperado el 08 de Julio de 2021, de http:// scielo.sld.cu/scielo.php?pid=S2307$21132017000300005 \&$ script $=$ sci $_{-}$ arttext\&tlng=pt

Barba Maggi, M. A., De la Calle Andrade, L. J., Fernández Villacrés, G. E., Gualpa Jaramillo, G. G., \& Calderón Barba, E. V. (2019). Aplicaciones móviles con tecnología Android para el aprendizaje de Medicina en las universidades UNIANDES, UNACH, UTB y UCACUE. En INVESTIGACIÓN Y ACADEMIA: LA VISIÓN DESDE LA UNIVERSIDAD
ECUATORIANA. COLECCIÓN INTERNACIONAL APROPIACIÓN, GESTIÓN Y USO EDIFICADOR DEL CONOCIMIENTO. (Vol. 6, págs. 4162). Red Iberoamericana de Pedagogía REDIPE. Recuperado el 7 de Julio de 2021, de https://redipe.org/wp-content/ uploads/2019/02/Libro-Investigacion$y$-academia-la-vision-desde-launiversidad-ecuatoriana.pdf

Barba, M. A., De la Calle, L., \& Fernández, G. (2018). Aplicación de objetos de aprendizaje 3D en Neuroanatomía. En N. Samaniego, \& C. Radicelli (Edits.), Aplicación, Experiencias y Desafíos de las TIC en el Ecuador (Primera ed., págs. 55-96). GCPI UNACH. Recuperado el 06 de Julio de 2021, de https:// ticec2018.cedia.edu.ec/dmdocuments/ TICEC/2018/PUBLICACIONES/TICS-E

Barba, M. A., De la Calle, L., Calderón, É. V., \& Ramos, Y. (Diciembre de 2019). Capacitación en el diseño de objetos de aprendizajepara la actualización en recursos didácticos con sustento en la Tic. Pro Sciences Revista de Producción, Ciencias e Investigación, 3(29), 9-19. doi:https://doi.org/10.29018/issn.25881000vol3iss29.2019pp9-19

Barroso, J., Cabero, J., \& Gutiérrez, J. J. (2018). La producción de objetos de aprendizaje en realidad aumentada por estudiantes universitarios. Grado de aceptación de esta tecnología y motivación para su uso. Revista Mexicana de Investigación Educativa, 23(79), 1261-1283. Obtenido de http://www. scielo.org.mx/scielo.php?pid=S140566662018000401261\&script=sci_arttext

Cabanillas, J., Luengo, R., \& Carvalho, J. (Diciembre de 2019). Análisis de los objetos de aprendizaje 
y de la percepción docente del campus virtual de la Universidad de Extremadura. International Journal of Information Systems and Software Engineering for Big Companies, 41-61. Recuperado el 07 de Julio de 2021, de https://dialnet.unirioja.es/servlet/ articulo?codigo $=7218461$

Cárdenas, I., \& Cáceres, M. L. (2019). Las generaciones digitales y las aplicaciones móviles como refuerzo educativo. Revista Metropolitana de Ciencias Aplicadas, 2(1), 25-31. Recuperado el 09 de Julio de 2021, de http://remca. umet.edu.ec/index.php/REMCA

Consejo de Educación Superior. (25 de Octubre de 2017). RPC-SO-39-No.726-20 17. Recuperado el 09 de Julio de 2021, de Gaceta Oficial del CES: http:// gaceta.ces.gob.ec/inicio.html?id_ documento $=229780$

Díaz, K., Miguel, V., Landaeta, I., Ortiz, M., \& Fernández, M. (2014). Satisfacción Estudiantil con el uso de Estrategias Instruccionales Apoyadas en Tic para el Aprendizaje de Bioquímica. Docencia Universitaria, $X V(2)$, 3-16. Recuperado el 09 de Julio de 2021, de http://190.169.30.98/ojs/index.php/rev docu/article/view/8467

Diez, E., \& Morales, R. A. (Diciembre de 2020). Codiseño de Objetos de Aprendizaje OA como estrategia de capacitación a docentes de Educación Superior. EDUTEC. Revista Electrónica de Tecnología Educativa(74), 114126. doi:https://doi.org/10.21556/ edutec.2020.74.1765

Fernández Villacrés, G. E., Arcos Naranjo, G. A., Barba Maggi, M. A., De la Calle Andrade, L. J., \& Gualpa Jaramillo,
G. G. (2016). Objetos de aprendizaje para la enseñanza de Telemedicina en las Universidades: UNIANDES, UTA y UNACH. UNIANDES EPISTEME: Revista de Ciencia, Tecnología e Innovación, 3(3), 372-383. Recuperado el 08 de Julio de 2021, de https://dialnet.unirioja.es/servlet/ articulo?codigo $=6756323$

Fombona , J., Pascual, M. A., \& VásquezCano, E. (2020). M-Learning en niveles iniciales, rasgos didácticos de las APPS educativas. Campus virtuales, 9(1), 1727. Recuperado el 08 de Julio de 2021, de http://www.uajournals.com/ojs/index. php/campusvirtuales/article/view/385

García, J., Fernández, J., Sánchez, A. J., \& Grimaldi, M. (2017). Gamificación y aplicaciones móviles para emprender: una propuestaeducativa en la enseñanza superior. International Journal of Educational Research and Innovation (IJERI), 8, 248-259. Recuperado el 08 de Julio de 2021, de https://rio.upo.es/ xmlui/handle/10433/5306

Gironés, J. T. (2013). El gran libro de Android (Tercera ed.). Barcelona, España: Marcombo S.A. Ediciones Técnicas. Recuperado el 08 de Julio de 2021, de https://books.google.com.ec/ books?id=K9hnCJ_NGq4C\&pg= PT22\&lpg $=$ PT22\&dq $=\% E 2 \% 80 \%$ A2\%09Alta+calidad+de+gr\% C3\%A1ficos+y+sonidos:+gr\%C3\% $A 1$ ficos+vector $\# v=$ onepage $\& q \& f=$ false

Maldonado, J. (2015). Desarrollo de un marco de análisis para la selección de metodologías de diseño de objetos de aprendizaje (OA) basado en criterios de calidad para contextos educativos específicos. Buenos Aires, Argentina: Universidad Nacional de 
la Plata. Recuperado el 08 de Julio de 2021, de http://sedici.unlp.edu.ar/ handle/10915/45063

Organización de las Naciones Unidas para la Educación, la Ciencia y la Cultura. UNESCO. (2021). WWW.UNESCO. ORG. Recuperado el 8 de Julio de 2021, de EDUCACIÓN SUPERIOR: https:// es.unesco.org/themes/educacionsuperior

Rodríguez Beltrán, N. M., Pardo Gómez, M. E., Izquierdo La O, J. M., \& Berenguer Gouarnaluses, J. A. (2018). GestiónAcadémicaen la EducaciónMedica Superior a través de Entornos de Enseñanza Aprendizaje. Convención Internacional de Salud, CubaSalud 2018. Recuperado el 8 de Julio de 2021, de http://www. convencionsalud2018.sld.cu/index. php/connvencionsalud/2018/paper/

Velásquez, S. M., Monsalve, D. E., Zapata, M. E., Gómez, M. E., \& Ríos, J. P. (Enero-Junio de 2019). Pruebas a aplicaciones móviles: avances y retos. Lámpsakos, 21, 39-50. doi:DOI: https:// doi.org/10.21501/21454086.2983

Velázquez, C., Álvarez, F., Muñoz, J., Cardona, P., Silva, A., Hernández, Y., \& Cechinelc, C. (Enero de 2014). Un Estudio de la Satisfacción Obtenida con el Uso de Objetos de Aprendizaje. Conference: IX Conferencia Latinoamericana de Objetos de Aprendizaje y Tecnolog'ias para el Aprendizaje (LACLO2014), (págs. 249257). Manizales. Recuperado el 09 de Julio de 2021, de https://www.semanticscholar.org/paper/ Un-Estudio-de-la-Satisfacci\%C3\%B3nObtenida-con-el-Uso-C\%C3\% A9sarVel\%C3\% A1zquez-Francisco\%C3\%81lvarez d30c437e146eb95cba7e9d58763825d $1 \mathrm{fb} 6 \mathrm{a} 39 \mathrm{bf}$ 